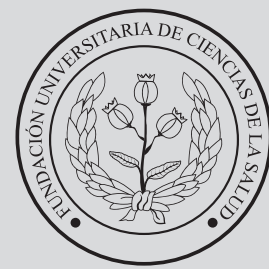

FUCS
Repro

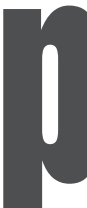

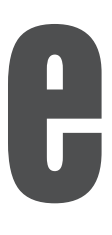

\title{
Precisión del pronóstico de la propagación del COVID-19 en Colombia
}

Jorge Enrique Díaz-Pinzón ${ }^{a}$

\section{Accuracy of COVID-19 propagation forecast in Colombia}

${ }^{a}$ Ingeniero. Magister en Gestión de la Tecnología Educativa, Especialista en Administración de la Informática Educativa. Docente de matemáticas e Investigador, Secretaría de Educación de Soacha, Cundinamarca.

\section{R E S U M E N}

Introducción: el nuevo Coronavirus (COVID-19) ha sido clasificado por la Organización Mundial de la Salud como una emergencia en salud pública de importancia internacional (ESPII). Se han reconocido casos en todos los continentes. El 6 de marzo de 2020 se confirmó el primer caso en Colombia. Objetivo: presentar la precisión de un pronóstico de la dinámica de transmisión del COVID-19 en Colombia. Metodología: para desarrollar la investigación se utilizó la base de datos de las personas infectadas con el Covid-19, esta información corresponde al período 6 de marzo al 14 de abril de 2020. Para su análisis de predicción se manejó el método modelo de Brown, utilizando el paquete estadístico SPSS v.25. Resultados: se apreció que el error de pronóstico fue muy bajo y correspondió al MAPE (error porcentual medio absoluto), con un 0,03\%, seguido del MAD (desviación media absoluta), con un valor de 0,95, es decir que en ambos casos la predicción obtuvieron un alto grado de confiabilidad. Conclusiones: el uso de modelación matemática se ha desarrollado en grado representativo en las últimas décadas y son de gran impulso para ilustrar escenarios eficaces de prevención y control de enfermedades infectocontagiosas.

Palabras clave: Covid-19, pronóstico, Transmisión de enfermedad infecciosa; Infección por Coronavirus.

Historia del artículo:

Fecha recibido: abril 15 de 2020

Fecha aceptado: abril 30 de 2020

\section{INFORMACIÓN DEL ARTÍCULO}

Autor para correspondencia.

Ing. Jorge Enrique Díaz Pinzón jediazp@unal.edu.co
DOI

10.31260/RepertMedCir.01217372.1045 
Introduction: the new Coronavirus (COVID-19) has been declared by the World Health Organization as a public health emergency of international concern (PHEIC). Cases have been reported on all continents. The first case was confirmed in Colombia on March 6 2020. Objective: to present the accuracy of a forecast of the dynamics of COVID-19 in Colombia. Methodology: the database including people infected with Covid-19 was used to develop the research. This information corresponds to the period between March 6 and April 14 2020. The Brown's model method was used for the predictive analysis in the SPSS v.25 statistical package. Results: it was observed that the prediction error value was very low and corresponds to a mean absolute percentage error (MAPE) of $0.03 \%$, followed by a mean absolute deviation (MAE) of 0.95 , hence, in both cases the quality of prediction had a high degree of reliability. Conclusions: in the last decades, the use of mathematical models has been developed at a representative degree, providing a major impetus for illustrating effective scenarios of infectious disease prevention and control.

Key words: Covid-19, prognosis, infectious disease transmission; Coronavirus infection

(C) 2020 Fundación Universitaria de Ciencias de la Salud - FUCS. This is an open access article under the CC BY-NC-ND license (http://creativecommons.org/licenses/by-nc-nd/4.0/).

\section{INTRODUCCIÓN}

Los coronavirus son una amplia familia de virus que logran causar enfermedades tanto en animales como en humanos. En los humanos, se sabe que varios coronavirus ocasionan infecciones respiratorias que consiguen ir desde el resfriado común hasta enfermedades más complicadas como el síndrome respiratorio de Oriente Medio (MERS) y el síndrome respiratorio agudo severo (SARS). El coronavirus que se ha manifestado más recientemente causa la enfermedad por coronavirus COVID-19. ${ }^{1}$

Según el Ministerio de Salud y Protección Social ${ }^{2}$ los coronavirus (CoV) son virus que brotan habitualmente en diferentes áreas del mundo y causan infección respiratoria aguda (IRA) es decir gripa, que pueden llegar a ser leve, moderada o grave.

El nuevo Coronavirus (COVID-19) ha sido clasificado por la Organización Mundial de la Salud como una emergencia en salud pública de importancia internacional (ESPII). Se han reconocido casos en todos los continentes y el 6 de marzo se confirmó el primer caso en Colombia. La infección se evidencia cuando una persona enferma tose o estornuda y expulsa partículas del virus que entran en contacto con otras personas.

En diciembre 2019 surgió una asociación de casos de neumonía en la ciudad de Wuhan (provincia de Hubei, China), con una muestra común a un mercado mayorista de mariscos, pescado y animales vivos. El 7 de enero 2020 las autoridades chinas establecieron como agente promotor del brote un nuevo virus de la familia coronaviridae, que ulteriormente fue designado SARS-CoV-2. La secuencia genética fue compartida por las autoridades chinas el 12 de enero. La enfermedad ocasionada por este nuevo virus se ha designado por consenso internacional COVID-19. El Comité de Emergencias del Reglamento Sanitario Internacional (RSI, 2005) manifestó el brote como una emergencia de salud pública de importancia internacional (ESPII) en su reunión del 30 de enero de 2020. Posteriormente el 11 de marzo de 2020 la OMS lo consideró como una pandemia global. ${ }^{3}$

En Colombia, el Instituto Nacional de Salud (INS) notifica diariamente al Ministerio de Salud las cifras de casos confirmados acumulados de COVID-19: total casos, casos en hospitalizaciones, ingreso en UCI, fallecidos y casos recuperados.

A pesar de las múltiples incertidumbres que a nivel biológico, clínico y epidemiológico subsisten en relación con este nuevo virus, lo que ya parece claro es que cada país ha respondido o está respondiendo a la misma amenaza con diferentes medidas y/o con una temporización diferente. Este hecho hace que las curvas epidemiológicas de los países afectados se estén comportando de manera distinta y que el costo social y económico de las respectivas respuestas pueda ser diferente. ${ }^{4}$

Según Montesinos ${ }^{5}$, el empleo de modelos matemáticos para enfermedades infecciosas ha aumentado en grado significativo en los años anteriores debido a que suministran información ventajosa para tomar decisiones, y establecer medidas activas en el control o erradicación de una enfermedad infecciosa. Estos modelos son muy ventajosos porque sujetan propiedades esenciales de la dispersión de una enfermedad de una forma sintética.

El pronóstico atañe a una predicción de un evento de interés (EI) después de su inicio. El término, hace referencia a posibles resultados de un EI y a la frecuencia con la que se puede esperar que se originen. ${ }^{6}$

El objetivo de este trabajo de investigación es presentar la precisión de un pronóstico de la dinámica de transmisión del COVID-19 en Colombia; el método utilizado para hallar el pronóstico fue el modelo lineal de Brown teniendo en cuenta el registro de la primera persona infectada el 6 de marzo 2020 hasta el 13 de abril 2020. 


\section{METODOLOGÍA}

\section{Tendencia lineal de Brown}

Este modelo es apropiado para las series con una tendencia lineal y sin estacionalidad. Sus parámetros de suavizado son el nivel y la tendencia, que se admiten iguales. Por ello, el modelo de Brown es un caso específico del modelo de Holt. El modelo de suavizado exponencial de Brown es muy análogo a un modelo ARIMA con cero órdenes de auto regresión, dos órdenes de distinción y dos órdenes de media móvil, con el coeficiente para el segundo orden de media móvil igual al cuadrado de la mitad del coeficiente de primer orden. ${ }^{7}$

Este método radica en realizar dos suavizaciones exponenciales, a partir de las cuales se alcanzará el valor estimado, o pronóstico que indagamos realizar, mediante un cálculo realizado con una expresión sencilla. La primera se emplea a los valores observados en la serie de tiempo y la segunda a la serie mitigada obtenida mediante la primera atenuación. Debido a que los valores calculados al realizar las dos primeras atenuaciones no son los datos considerados a obtener, es decir, que formarán las inferencias de los valores que se espera que tome la serie de tiempo en el futuro cercano, emplearemos una notación distinta a la de la expresión final con la cual se calculan los valores que componen en realidad el pronóstico. ${ }^{8,9}$

Este es el modelo más ventajoso para el pronóstico a corto plazo, se identifica porque busca mitigar los valores picos de la función por medio de un coeficiente denominado Alfa " $a$ ". ${ }^{10}$

El objetivo de los métodos de serie de tiempo es manifestar un patrón en los datos históricos y luego extrapolarlo hacia el futuro; el pronóstico se fundamenta solo en valores pasados de la variable que tratamos de predecir. ${ }^{11} \mathrm{~A}$ continuación, se presenta la formulación del método de Brown:

$$
\begin{aligned}
& \mathrm{St} 1=(a \times \mathrm{Xt})+[(1-a) \times \mathrm{St}-1] \\
& \mathrm{St} 2=(a \times \mathrm{St})+[(1-a) \times \mathrm{St}-1] \\
& \mathrm{at}=\mathrm{St} 1+(\mathrm{St} 1-\mathrm{St} 2)=\mathrm{St} 1-\mathrm{St} 2 \\
& \mathrm{bt}=a /(1-a)(\mathrm{St} 1-\mathrm{St} 2) \\
& \mathrm{Ft}+\mathrm{m}=\mathrm{at}+\mathrm{btm}
\end{aligned}
$$

Donde:

- Stl = Valor del suavizamiento exponencial simple al finalizar el periodo $t$

- St2 = Valor del suavizamiento exponencial doble al finalizar el periodo $\mathrm{t}$

- at = Ajuste de la serie al finalizar el periodo $t$

- $b t=$ Ajuste de tendencia al finalizar el periodo $t$

- $\boldsymbol{a}=$ Constante de aislamiento

- $\mathrm{m}=$ Varia desde 1 hasta $\mathrm{m}, \mathrm{y}$ determina el número de pronósticos que se deseen

- $\mathrm{Ft}+\mathrm{m}=$ Pronostico en el periodo $\mathrm{t}+\mathrm{m}$

\section{Medición del error en pronósticos}

Persistentemente se van a presentar errores en el cálculo de un pronóstico. En la práctica, se pretende minimizar ambos tipos de errores optando por el mejor método de pronóstico, y es por eso que existe la medición del error en pronósticos. Las medidas de error de pronóstico calculadas para un solo método en un solo período de tiempo carecen de significado. Su utilidad reside cuando comparamos las medidas de error con las medidas de otros métodos de pronóstico o con otros períodos de tiempo. ${ }^{12}$ A continuación, describiremos varias medidas de error y sus correspondientes fórmulas que utilizaremos en la precisión del pronóstico de acuerdo con Betancourt. $^{12}$

\section{Suma acumulada de errores de pronóstico (CFE)}

Es la medida más primordial de todas y es la que da origen a las demás. Es la suma acumulada de los errores de pronóstico.

$$
\mathrm{CFE}=\sum \text { Error de pronóstico }
$$

\section{Desviación media absoluta (MAD)}

Mide la dispersión del error de pronóstico o, dicho de otra manera, la medición del tamaño del error en unidades. Es el valor absoluto de la diferencia entre el riesgo real y el pronóstico, dividido sobre el número de periodos.

$$
\mathrm{MAD}=\frac{\sum \mid \text { Real-Pronóstico }}{\mathrm{n}}
$$

\section{Error cuadrático medio (MSE)}

Al igual que la DAM, el MSE es una medida de dispersión del error de pronóstico, no obstante, esta medida maximiza el error al elevar al cuadrado, infligiendo aquellos periodos donde la diferencia fue más alta a comparación de otros. En consecuencia, se recomienda el uso del MSE para periodos con desviaciones pequeñas.

$$
\text { MSE }=\frac{\sum \text { Error de pronóstico }{ }^{2}}{n}
$$

\section{Error porcentual medio absoluto (MAPE)}

El MAPE entrega la desviación en términos porcentuales $\mathrm{y}$ no en unidades como las anteriores medidas. Es el promedio del error absoluto o diferencia entre el riesgo real y el pronóstico, indicado como un porcentaje de los valores reales.

$$
\text { MAPE }=\frac{\frac{\sum_{\mathrm{i}=1}^{\mathrm{n}} 100 \mid \text { Real }_{\mathrm{i}}-\text { Pronóstico }_{\mathrm{i}} \mid}{\text { Real }_{\mathrm{i}}}}{\mathrm{n}}
$$

\section{Señal de rastreo}

La señal de rastreo es una medida de desempeño que permite medir la desviación del pronóstico respecto a 
variaciones en la demanda. Las señales de rastreo positivas indican que el riesgo real es mayor que el pronóstico. Las señales de rastreo negativas indican que la demanda es menor que el pronóstico. Los errores negativos y positivos deben equilibrarse entre sí para que la señal de rastreo se centre muy cerca de cero. (De Antonio)

$$
\text { Señal de rastreo }=\frac{\text { CFE }}{\text { MAD }}
$$

Se realizó un estudio descriptivo de análisis de series temporales correspondiente al período 6 de marzo 2020 a 14 de abril 2020 en Colombia, para obtener una precisión del pronóstico de contagio por COVID-2019. Las fuentes de información empleadas estuvieron constituidas por el conjunto de series cronológicas obtenidas del Ministerio de Salud y Protección Social.

Las características de la información disponible permitieron evaluar la calidad de las series y el aspecto necesario para poder comenzar el proceso de predicción. Se consideró al conjunto de las series como suficientemente consistente para realizar el análisis. Se decidió utilizar la información de casos confirmados por el contagio con COVID-19 y que se calcularon en 2979 personas afectadas, hombres 1529 y mujeres 1450. Se deseaba analizar la precisión del pronóstico versus el riesgo real de contagio en el período 6 de marzo 2020 a 14 de abril 2020. Para su análisis de predicción se manejó el método de predicción modelo de Brown, utilizando el paquete estadístico SPSS v.25.

Ahora para llevar a cabo la precisión del pronóstico que es el grado de cercanía entre la expresión de cantidad y el valor real de ésta, se emplearon métricas para calcular la precisión de los pronósticos MAE (error absoluto medio), MAPE (error absoluto medio relativo), MSE (error cuadrático medio) y CFE (suma acumulada de errores de pronóstico).

\section{RESULTAD OS}

En la (tabla 1) se muestran los diferentes errores con la información de contagio real y pronóstico del COVID-19, durante el período 6 de marzo a 14 de abril 2020, con la información de 40 días de observación. Se aprecia que el error de pronóstico fue muy bajo y correspondió al MAPE (error porcentual medio absoluto) con un $0,03 \%$, seguido del MAD (desviación media absoluta) con un valor de 0,95 es decir que en ambos casos la predicción tiene un alto grado de confiabilidad.

En la (figura 1) se describe como la línea de contagio real de color azul y la línea de pronóstico de color naranja son muy similares entre sí, en la mayoría del recorrido permanecen superpuestas una con otra, confirmando los errores mínimos de la (tabla 1), es decir el pronóstico se ajusta al contexto de contagio real de pacientes con COVID-19.

\section{El coeficiente de determinación ajustado $\left(\mathbf{R}^{2}\right)$}

El $\mathrm{R}^{2}$, coeficiente de determinación nos indica en qué medida los datos se ajustan al modelo seleccionado, tendencia lineal de Brown, en este caso el $\mathrm{R}^{2}=0,998$, y si lo convertimos a porcentaje se va obtener un $99.8 \%$, esto significa que los datos se ajustan en un $99,8 \%$ al modelo seleccionado.

\section{CONCLUSIONES}

El modelo de Brown nos ilustra un escenario posible de la enfermedad, lo cual a la luz de los resultados no es nada halagador para Colombia, por lo tanto, se hace necesario continuar con el aislamiento para que se genere una estabilización de la enfermedad. Esta predicción puede cambiar si todos los colombianos somos responsables en guardar la cuarentena.

Este modelo analizado no tiene en cuenta el crecimiento hasta alcanzar un 'pico', y es fundamental para el sistema sanitario saber cuándo se va a llegar, por lo tanto, es necesario realizar un monitoreo permanente para esclarecer el futuro de la enfermedad.

Los alisamientos exponenciales establecen métodos muy simples y la posibilidad de ponderar las observaciones a través de las constantes de suavizamiento le otorga gran poder para la predicción. Ofrecer pronósticos, que brinden cierto grado de confianza, acerca de la evolución futura de las causas de muerte en un horizonte de tiempo determinado favorece a alcanzar una mejor preparación del sistema de salud para su enfrentamiento. ${ }^{9}$ Además, la utilización de modelos de predicción de comparativamente fácil construcción como los obtenidos con alisamientos exponenciales en este estudio, logran resultar muy útiles para la vigilancia de eventos de salud diversos ya que admiten a las autoridades sanitarias el conocimiento previo que facilita en gran medida la toma de decisiones oportunas. ${ }^{9}$

Se apreció que el error de pronóstico fue muy bajo, y correspondió al MAPE (error porcentual medio absoluto), con un 0,03\%, seguido del MAD (desviación media absoluta), con un valor de 0,95 , es decir que en ambos casos la predicción obtuvo un alto grado de confiabilidad.

El autor recomienda realizar otro tipo de análisis con otros modelos matemáticos por ejemplo el modelo SIR es uno de los modelos epidemiológicos más simples e idóneos para capturar muchas de las características típicas de los brotes epidémicos. El nombre del modelo procede de las iniciales $\mathrm{S}$ (población susceptible), I (población infectada) y R (población recuperada). El modelo concierne las variaciones las tres poblaciones (susceptible, infectada y recuperada) a través de la tasa de infección y el período infeccioso promedio. ${ }^{13,14}$

Consecuentemente, es vital identificar una población objetivo en la que las predicciones que convenzan una 
Tabla 1. Error de pronóstico para el COVID-19

\begin{tabular}{|c|c|c|c|c|c|c|}
\hline Periodo & Contagio Real & Pronóstico & Error de pronóstico & $\begin{array}{l}\text { Desviación absoluta } \\
\text { media (MAD) }\end{array}$ & $\begin{array}{l}\text { Error cuadrático medio } \\
\text { (MSE) }\end{array}$ & $\begin{array}{c}\text { Error porcentual absoluto } \\
\text { medio (MAPE) }\end{array}$ \\
\hline 1 & 1 & 1 & 0,00 & 0,00 & 0,00 & $0,00 \%$ \\
\hline 2 & 1 & 1 & 0,00 & 0,00 & 0,00 & $0,00 \%$ \\
\hline 3 & 1 & 1 & 0,00 & 0,00 & 0,00 & $0,00 \%$ \\
\hline 4 & 3 & 1 & 2,00 & 2,00 & 4,00 & $66,67 \%$ \\
\hline 5 & 3 & 4 & $-1,00$ & 1,00 & 1,00 & $33,33 \%$ \\
\hline 6 & 9 & 4 & 5,00 & 5,00 & 25,00 & $55,56 \%$ \\
\hline 7 & 13 & 13 & 0,00 & 0,00 & 0,00 & $0,00 \%$ \\
\hline 8 & 16 & 17 & $-1,00$ & 1,00 & 1,00 & $6,25 \%$ \\
\hline 9 & 24 & 19 & 5,00 & 5,00 & 25,00 & $20,83 \%$ \\
\hline 10 & 45 & 30 & 15,00 & 15,00 & 225,00 & $33,33 \%$ \\
\hline 11 & 57 & 61 & $-4,00$ & 4,00 & 16,00 & $7,02 \%$ \\
\hline 12 & 75 & 71 & 4,00 & 4,00 & 16,00 & $5,33 \%$ \\
\hline 13 & 102 & 91 & 11,00 & 11,00 & 121,00 & $10,78 \%$ \\
\hline 14 & 128 & 125 & 3,00 & 3,00 & 9,00 & $2,34 \%$ \\
\hline 15 & 175 & 153 & 22,00 & 22,00 & 484,00 & $12,57 \%$ \\
\hline 16 & 210 & 214 & $-4,00$ & 4,00 & 16,00 & $1,90 \%$ \\
\hline 17 & 240 & 247 & $-7,00$ & 7,00 & 49,00 & $2,92 \%$ \\
\hline 18 & 306 & 272 & 34,00 & 34,00 & 1156,00 & $11,11 \%$ \\
\hline 19 & 378 & 360 & 18,00 & 18,00 & 324,00 & $4,76 \%$ \\
\hline 20 & 470 & 444 & 26,00 & 26,00 & 676,00 & $5,53 \%$ \\
\hline 21 & 491 & 535 & $-62,00$ & 62,00 & 3844,00 & $12,63 \%$ \\
\hline 22 & 539 & 553 & 4,00 & 4,00 & 16,00 & $0,74 \%$ \\
\hline 23 & 608 & 584 & 24,00 & 24,00 & 576,00 & $3,95 \%$ \\
\hline 24 & 702 & 668 & 34,00 & 34,00 & 1156,00 & $4,84 \%$ \\
\hline 25 & 798 & 785 & 13,00 & 13,00 & 169,00 & $1,63 \%$ \\
\hline 26 & 906 & 890 & 16,00 & 16,00 & 256,00 & $1,77 \%$ \\
\hline 27 & 1065 & 1.009 & 56,00 & 56,00 & 3136,00 & $5,26 \%$ \\
\hline 28 & 1161 & 1.204 & $-43,00$ & 43,00 & 1849,00 & $3,70 \%$ \\
\hline 29 & 1267 & 1.274 & $-7,00$ & 7,00 & 49,00 & $0,55 \%$ \\
\hline 30 & 1406 & 1.374 & 32,00 & 32,00 & 1024,00 & $2,28 \%$ \\
\hline 31 & 1485 & 1.533 & $-48,00$ & 48,00 & 2304,00 & $3,23 \%$ \\
\hline 32 & 1579 & 1.582 & $-3,00$ & 3,00 & 9,00 & $0,19 \%$ \\
\hline 33 & 1780 & 1.673 & 107,00 & 107,00 & 11449,00 & $6,01 \%$ \\
\hline 34 & 2054 & 1.942 & 112,00 & 112,00 & 12544,00 & $5,45 \%$ \\
\hline 35 & 2223 & 2.291 & $-68,00$ & 68,00 & 4624,00 & $3,06 \%$ \\
\hline 36 & 2473 & 2.420 & 53,00 & 53,00 & 2809,00 & $2,14 \%$ \\
\hline 37 & 2709 & 2.702 & 7,00 & 7,00 & 49,00 & $0,26 \%$ \\
\hline 38 & 2776 & 2.944 & $-168,00$ & 168,00 & 28224,00 & $6,05 \%$ \\
\hline 39 & 2852 & 2.904 & $-52,00$ & 52,00 & 2704,00 & $1,82 \%$ \\
\hline \multirow[t]{2}{*}{40} & 2979 & 2.941 & 38,00 & 38,00 & 1444,00 & $1,28 \%$ \\
\hline & & Suma de errores & 173,00 & 1101,00 & 79665,00 & $345,28 \%$ \\
\hline
\end{tabular}

\begin{tabular}{l|c} 
CFE & $\mathbf{3 8 , 0 0}$ \\
\cline { 2 - 2 } MAD & $\mathbf{0 , 9 5}$ \\
\hline MSE & $\mathbf{3 6 , 1 0}$ \\
\hline MAPE & $\mathbf{0 , 0 3 \%}$ \\
\hline Señal de rastreo & $\mathbf{4 0 , 0 0}$ \\
\hline
\end{tabular}

necesidad clínica, y un conjunto de datos representativo (preferiblemente que incluya pacientes consecutivos) en el que se pueda desarrollar y validar el modelo de predicción. En lugar de desarrollar y restaurar las predicciones en su entorno local, los datos de participantes individuales de múltiples países y sistemas de salud deberían permitir una mejor comprensión de la generalización e implementación de modelos de predicción en otros entornos y poblaciones. ${ }^{15}$

La inestabilidad en las frecuencias relativas de los resultados señalados presenta un reto importante para el modelador de predicciones. Un modelo de predicción 
aplicado en un entorno con una frecuencia relativa desigual del resultado podría originar predicciones mal calibradas ${ }^{16}$ y podría precisar actualizarse antes de que consiga aplicarse de forma segura en ese nuevo entorno. ${ }^{1718}$

\section{CONFLICTO DE INTERÉS}

El autor declara ningún conflicto de intereses.

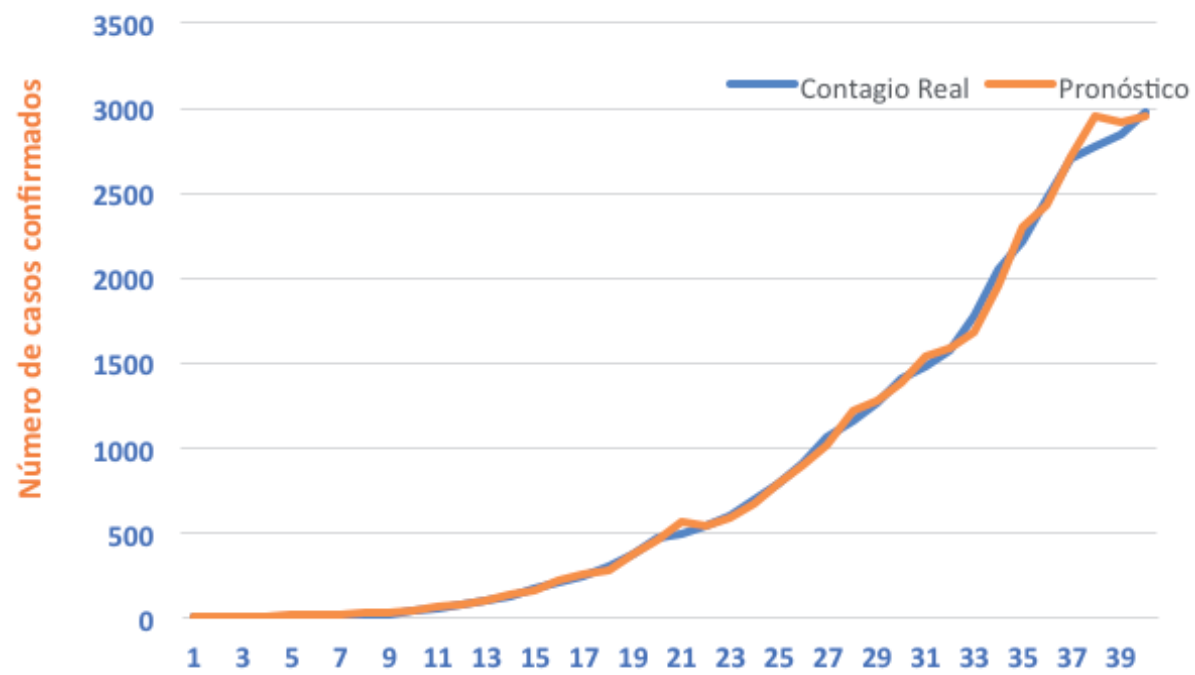

\section{Días desde que se registro el primer caso}

Figura 1. Contagio real versus pronóstico

\section{REFERENCIAS}

1. Organización Mundial de la Salud. OMS. Preguntas y respuestas sobre la enfermedad por coronavirus (COVID-19) [Internet]. 2020 [citado 2020 marzo 26]. Recuperado de: https://www.who.int/es/ emergencies/diseases/novel-coronavirus-2019/advice-for-public/ q-a-coronaviruses

2. Ministerio de Salud y protección Social. Orientaciones para el tamizaje de viajeros procedentes de zonas con circulación del nuevo coronavirus (covid-19) [Internet]. 2020 [citado 2020 marzo 26]. Recuperado de: https://www.minsalud.gov.co/sites/rid/Lists/ BibliotecaDigital/RIDE/VS/asif04-guia-tamizaje-poblacionalpuntos-entrada-coronavirus.pdf

3. ISCIII. Informe sobre la situación de COVID-19 en España. 2020 [Internet].2020[citado2020marzo26]. Recuperadode:https://www. isciii.es/QueHacemos/Servicios/VigilanciaSaludPublicaRENAVE/ EnfermedadesTransmisibles/Paginas/InformesCOVID-19.aspx

4. Chaccour, C. COVID-19: Cinco respuestas de salud pública diferentes ante la epidemia [Internet]. 2020 [citado 2020 marzo 26]. Disponible en: https://www.isglobal.org/healthisglobal/-/ custom-blog-portlet/covid-19-cinco-respuestas-de-salud-publicadiferentes-ante-la-epidemia/2877257/0

5. Montesinos, O. Hernández, C. Modelos matemáticos para enfermedades infecciosas [Internet]. 2020 [citado $2020 \mathrm{mar}$ 26]. Recuperado de: https://www.scielosp.org/pdf/spm/2007. v49n3/218-226

6. Manterola, C; Santander, C; Otzen, T. Cómo valorar e interpretar un artículo sobre pronóstico. Rev Chil Cir. 2013;65(1):77-84. doi: http://dx.doi.org/10.4067/S0718-40262013000100015

7. IBM. Modelos personalizados de suavizado exponencial [Internet]. 2010 [citado 2020 marzo 26]. Recuperado de: https://www. ibm.com/support/knowledgecenter/es/SSLVMB_sub/statistics_ mainhelp_ddita/spss/trends/idh_idd_exp_smooth_crit.html

8. Modelos de pronóstico. Suavización Exponencial Doble Método de Brown Ajuste a la Tendencia [Internet]. 2020 [citado 2020 abril 04]. Recuperado de:http://modelosdepronosticos.info/metodo_ de_suavizacion_exponencial_doble_metodo_de_brown.html

9. Coutin, M, Gisele. Pronósticos de mortalidad por enfermedades no transmisibles seleccionadas. Rev Cubana Hig Epidemiol. 2008;46(3):1-14

10. Ramos, D. Bacca, A. Importancia de la planeación de la demanda en una empresa del sector industrial [Internet]. 2014 [citado 2020 abril 7]. Recuperado de: https://core.ac.uk/download/ pdf/143448212.pdf

11.Villareal, F. Introducción a los Modelos de Pronósticos [Internet]. 2016 [citado 2020 abril 7]. Recuperado de: https://www. matematica.uns.edu.ar/uma2016/material/Introduccion_a_los_ Modelos_de_Pronosticos.pdf 
12. Betancourt, D. F. Medición del error en pronósticos de demanda [Internet]. 2016 [citado 2020 abril 13]. Recuperado de: https:// ingenioempresa.com/medicion-error-pronostico/

13. 8Galindo-Uribarri, S; Rodríguez- Meza, M; Cervantes-Cota, J. Las matemáticas de las epidemias: caso México 2009 y otros. Ciencia Ergo Sum. 2009;20(3):238-246.

14. Navarro-Robles, E. Martínez-Matsushita, L; López-Molina, R; Fritz-Hernández, F; Flores-Aldana, B; Mendoza-Pérez, J. Modelo para estimación del comportamiento epidémico de la influenza A (HlNl) en México [Internet]. 2011 [citado 2020 abril 04]. Recuperado de: https://scielosp.org/article/rpsp/2012.v31n4/269274/

15. BMJ 2020. Modelos de predicción para el diagnóstico y pronóstico de la infección por covid-19: revisión sistemática y valoración crítica. [Internet]. 2020 [citado 2020 abril 23]. Recuperado de: https://www.bmj.com/content/369/bmj.m1328

16. Van Calster B, McLernon DJ, van Smeden M, Wynants L, Steyerberg EW. Calibration: the Achilles heel of predictive analytics. BMC medicine. 2019;17(1):230. http://dx.doi.org/10.1186 / s12916-0191466-7

17. Steyerberg E. Clinical prediction models. A practical approach to development, validation, and updating. United State: Springer; 2009.

18. Riley RD, Ensor J, Snell KI, Debray TP, Altman DG, Moons KG, et al. External validation of clinical prediction models using big datasets from e-health records or IPD meta-analysis: opportunities and challenges. BMJ. 2016;353:i3140. http://dx.doi.org/10.1136/ bmj. i3140 